\title{
Measurement of absolute energy scale of ECAL of DAMPE with geomagnetic rigidity cutoff
}

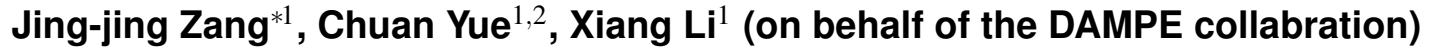 \\ $\dagger$ \\ ${ }^{1}$ Key Laboratory of Dark Matter and Space Astronomy, Purple Mountain Observatory, Chinese \\ Academy of Sciences, Nanjing 210008, China \\ ${ }^{2}$ University of Chinese Academy of Sciences, Beijing 100049, China \\ E-mail: zangjjepmo.ac.cn
}

\begin{abstract}
In this paper, we developed a method to determine absolute energy scale of DAMPE via measuring geomagnetic cutoff on cosmic ray electron and positron spectrum. The rigidity cutoff on cosmic ray electron and positron was calculated using IGRF-12 model and cosmic ray particle trajectory tracing code developed by Smart and Shea. Then we also measured cosmic ray electron and positron spectrum in MacIlwain $L$ bin $[1,1.14]$ based on over 425 days flight data of DAMPE. By directly comparing calculated geomagnetic cutoff with DAMPE measured result, we provide an estimation on absolute energy scale of DAMPE $1.25 \%$ higher than expected at about $13 \mathrm{GeV}$ energy with uncertainty about $\pm 1.75 \%$ (stat) $\pm 1.34 \%$ (sys).
\end{abstract}

35th International Cosmic Ray Conference - ICRC2017

10-20 July, 2017

Bexco, Busan, Korea

\footnotetext{
${ }^{*}$ Speaker.

${ }^{\dagger}$ The DAMPE mission is funded by the National Key Research and Development Program of China (No. 2016YFA0400200). In China the data analysis is supported in part by the strategic priority science and technology projects in space science of Chinese Academy of Sciences (No. XDA04040000), National Basic Research Program of China (No. 2013CB837000), National Natural Science Foundation of China (No. 11303105) and Young Elite Scientists Sponsorship Program by CAST (No. YESS20160196). In Europe the activities and the data analysis are supported by the Swiss National Science Foundation (SNSF), Switzerland; the National Institute for Nuclear Physics (INFN), Italy.
} 


\section{DAMPE detector introduction}

The Dark Matter Particle Explorer (DAMPE) is a cosmic ray space telescope with high energy resolution and extreme large dynamic range, which was launched on December 17, 2015, into a sun-synchronous orbit at 500km altitude with 97 degree inclination. DAMPE's main scientific goal is to probe high energy cosmic ray $e^{+} / e^{-}$and $\gamma$ from several $\mathrm{GeV}$ to more than $10 \mathrm{TeV}$ to precisely measure the spectrum structure and space distribution etc. DAMPE also aims at measuring flux of cosmic ray nucleus to near first "knee" range. To achieve those goals, DAMPE is equipped with a silicon tungsten tracker (STK) and a BGO crystal calorimeter (BGO) in the middle, covered with 82 plastic scintillator detector (PSD) in $\mathrm{x}-\mathrm{y}$ direction on the top to decide particle charge and 4 Boron-doped plastic scintillant plates on the bottom to identify neutrons generated in BGO to enhance e/p separation power. The sketch map of DAMPE can be found in Fig1. BGO calorimeter

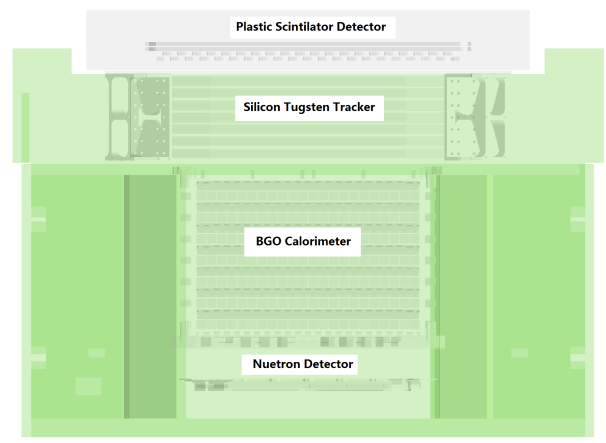

Figure 1: The sketch map of DAMPE detectors. Designed as high sensitive cosmic ray electron detector, from top to bottom, DAMPE is equipped with plastic scintillator detector (PSD), silicon tungsten tracker (STK), BGO crystal calorimeter (BGO) and neutron detector (NUD).

is consisted by 14 layers of a orthogonal array of BGO crystal bars with 31 radiation lengths in depth. For the requirement of large dynamic range, fluorescent light signal generated by particle is read out from both ends of crystal, each end is coupled with light attenuator with different light decay rate. By this design, BGO can measure deposited energy in two overlapping dynamic ranges, namely $2 \mathrm{MeV}-2 \mathrm{TeV}$ and $10 \mathrm{MeV}-5 \mathrm{TeV}$.

In DAMPE's flight data production pipe line, energy reconstruction of BGO is roughly separated into 2 steps: Pre-Reco and Reco. In the Pre-Reco progress, the Analog-to-Digital Converter (ADC) signals of BGO bars are converted into energy hits by an elaborate algorithm. This algorith$m$ is meant to equalize the response of each crystal read-out dynode and to convert the ADC signal to energy via the calibration constants derived from cosmic-ray proton MIP events and DAMPE's calibration electronics circuits. In procedure of Reco, energy hits are reconstructed to final energy by a series of correction algorithms, including side and back end energy leakage correction and dead zone correction[3]. These algorithms are all verified by beam test at CERN in 2014 and 2015[1]. A more complete description of these algorithms will be presented in section 3.

\section{Meaning of absolute energy scale measurement}

Absolute energy scale is a systematic bias on energy measurement. If DAMPE wants to pre- 
cisely measure spectral feature, it is very necessary to find a way to estimate it. During R\&D stage, an extensive beam test on EQM was performed at CERN's PS and SPS accelerator in 2014 and 2015. In principle, beam moment is well tuned by a series of magnets on beam line and can severed as benchmark of energy, but beam always impacts on DAMPE with fixed angle and position. It is impossible to tune an isotropic beam to mimic cosmic ray particles. Considering risks of shipping from China to Europe, beam test was not performed on FM (short for flight model). It is very difficult to precisely estimate the deference between EQM and FM. Therefore, it is unable to use beam moment as the reference of absolute energy scale. In Geophysics, it is well known that cosmic ray with certain rigidity will be bent back to space by geomagnetic force. This phenomenon causes a clear cutoff on spectrum of cosmic ray electron and positron ${ }^{1}$ at $10 \mathrm{GeV}$ [8]. Based on this kind of cutoff, Fermi-LAT successfully measured the absolute energy scale of their electromagnetic calorimeter with accuracy at about 2.5\% level[2] in several MacIlwain L[5] intervals.

\section{Energy Reconstruction method}

The sensitive matter of DAMPE's calorimeter is 308 BGO crystal bars (with dimensions $25 \mathrm{~mm} \times 25 \mathrm{~mm} \times 600 \mathrm{~mm}$ ), which are arranged in 14 layers, each with 22 carbon fiber honeycomb capsules spanning width of the layer. The capsules of neighbor layers are rotated by $90^{\circ}$ about the boresight of DAMPE, which make BGO CAL a 3D imaging calorimeter. Each crystal is read out from both ends with 3 dynodes of PMT, modifying signal with low, middle and high gains to cover three different overlapped energy ranges, which means one fluorescence signal especially for dozens $\mathrm{GeV}$ range can be measured at least four times( 2 ends \& 2 dynodes). By this design, the energy resolution can reach $1-2 \%[3]$ at dozens GeV. A major advantage of DAMPE's absolute energy scale measurement is that DAMPE has better energy resolution comparing with Fermi-LAT (about $7 \%$ energy resolution at $13 \mathrm{GeV}[7]$ ).

ADC counts collected from electronics mainly contain pedestal, random noise and signal. To regularly calibrate pedestals of all readout electronics in a short time interval, random trigger run is performed twice per orbit. Besides that, we also enabled MIPs calibration run at $-20^{\circ}$ to $20^{\circ}$ latitude to collect proton events penetrating all layers of BGO CAL with no shower and most probable values of these MIP spectra will be treated as an energy reference for each crystal. Comparing signals from electronics with these references could help us normalize raw ADC counts to times of MIPs. Since MIP events deposit relative small energy, and in consequence only high gain channels can extract MIP references. Signals from low and middle gain channels should be firstly converted to amount of high gain channels by gain ratios and then will be evaluated to times of MIPs. By frequently MIPs calibration, the variation of MIP energy scale can be kept within $0.5 \%$ during whole data-taken period, more details will be published in DAMPE on-orbit calibration paper.

Event energy reconstruction starts from accumulating energy deposition in each BGO crystal. A series of empirical formula[3] are used to correct energy deposited in insensitive matter and leakage from back and side. At last, absolute energy scale of MIPs will be determined by carefully on-orbit Monte Carlo simulation. Although verification of simulation shows a well agreement with

\footnotetext{
${ }^{1}$ DAMPE can not discriminate the sign of particle's charge, hereafter cosmic ray electron or CRE means cosmic ray electron and positron.
} 
flight data[3], proton MIPs events can only determine absolute energy scale at very low energy range (MPV of proton MIPs in BGO is $\sim 23 \mathrm{MeV}$ ), and so many factors can affect this kind of energy reconstruction at high energy range. Therefore, measurement of absolute energy scale is an essential job for energy or spectrum measurement.

\section{Electron sample selection (e/p separation)}

In this analysis, we used 425 days DAMPE data collected from Jan 2016 to Feb 2017. To efficiently reject "junk" events beyond the field of view (FoV) of DAMPE while keeping electronlike particles, a serious pre-selections are designed to select ąrgoodąś events with reliable track and good shower development in BGO-CAL. The event selection started from trigger logic requirement and energy range cut. We firstly select trigger logic for high energy electron and demanded energy of events should be in range $[400 \mathrm{MeV}, 150 \mathrm{GeV}]$ which is a little bit wider than final interesting energy range. Then we require that the STK reconstructs at least one good track in the FoV. To roughly reject side-impact events, we constructed two variables to describe lateral shower size in one layer and longitudinal size. If particles really penetrate whole DAMPE detectors from top to bottom, longitudinal size should be greater than lateral size. Besides that, we also set several requirements on number of BGO hits, energy ratio deposited in first 10 layers to total energy, shower max cell position to abandon low quality events. All selections mentioned above were well tuned by DAMPE MC simulation and beam test data which can efficiently reject background while keep most of electrons. At last, we got about 40M "good" events which are still proton dominated due to proton flux is 2-3 orders larger than electron flux at dozens GeVs.

\section{Background estimation}

\subsection{CR-hadron background}

The data sample after pre-selections is mixed with cosmic ray hadron. Based on different behavior between electromagnetic (EM) shower and hadronic shower, we developed two variables to separately describe transverse shower size (TS) and longitudinal shower size (LS). Technically, to maximize electron-hadron discrimination power, we combined these two variables into variable P, according to definition $P=\log (T S) * \cos \theta-\log (L S) * \sin \theta$, where $\theta$ is rotation angle in the coordinator of TS-LS. The typical distributions of variable $\mathrm{P}$ are shown in Fig2. We also plot same distribution from MC simulation in Fig2, result shows a well agreement between MC and flight data. Then template fitting was used to extracted hadron background ratio. Finally, the determination of CR-hadron contamination is performed for all energy bins. Fig2 shows the contamination are about $1 \%$ level at about a dozen $\mathrm{GeV}$ range and was kept less than $5 \%$ for all energy bins above $10 \mathrm{GeV}$. A major advantage of DAMPE's absolute energy scale measurement compared with Fermi-LAT is that DAMPE has higher e/p separation power which can suppress systematic uncertainty from hadron background contamination. It is also found that the contamination from hadron become severe with no matter energy increasing or decreasing. In low energy range, over $90 \%$ of particle's energy was deposited in only 4-5 BGO bars, the granularity of BGO ECAL are relative large comparing with small shower size which induce that lower precision on shower shape measurement. As a consequence, the electron-hadron suppression power becomes weak at very low 
energy range. At high energy range, the relative flux of proton with respect electron increases from less than 200 times higher to more than 800 times higher[4, 6], which causes more severe proton background contamination even if the e/p separation is better at high energy range.

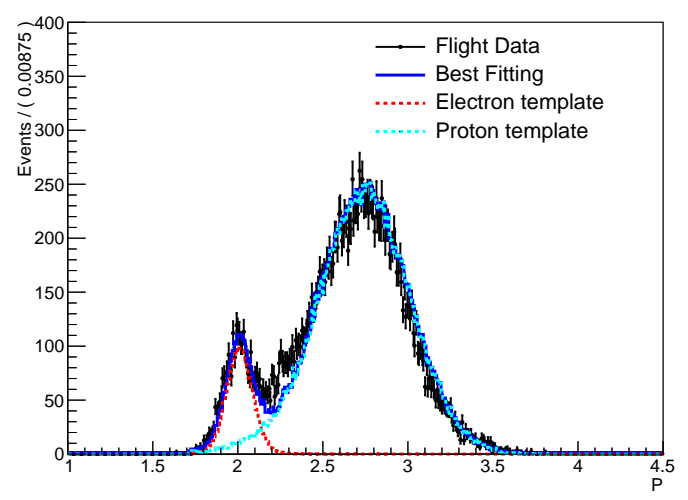

(a) bin 1

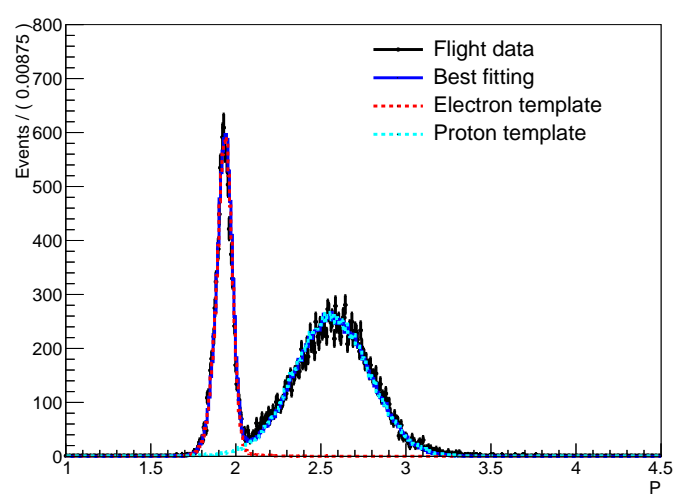

(c) bin19

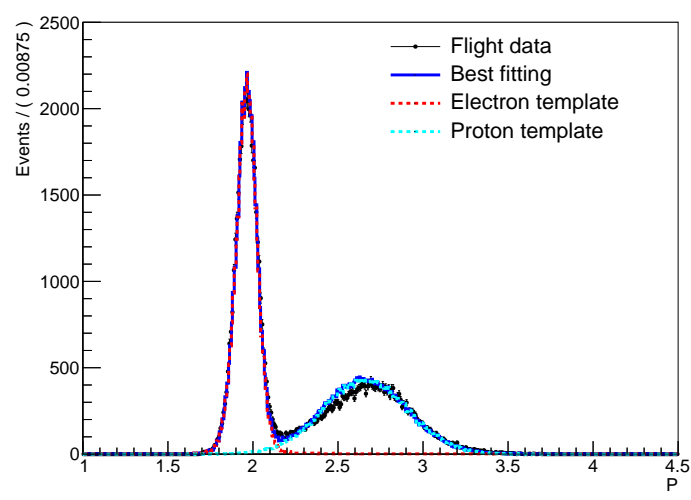

(b) bin8

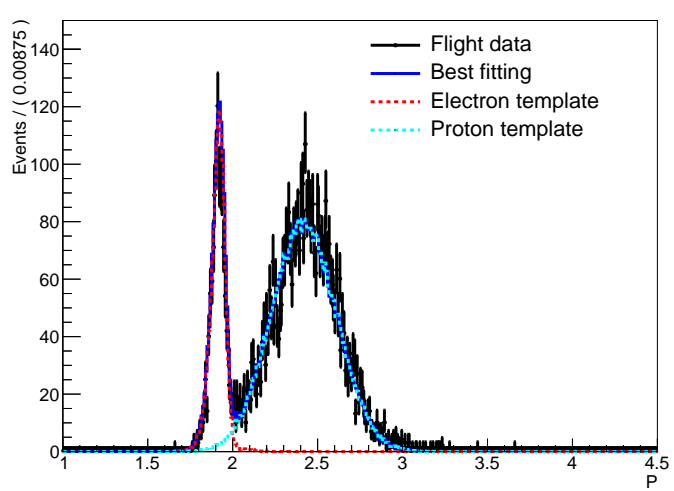

(d) bin 30

Figure 2: Typical distribution of variable $\mathrm{P}$ at deposited energy in ranges (a) $[8,8.7] \mathrm{GeV}$, (b) [14.4, 15.7] $\mathrm{GeV}$, (c)[36.4, 39.6] GeV, (d) $[91.9,100.0] \mathrm{GeV}$. The black dot with error bar is flight data distribution, red/blue dashed line is electron/proton MC template and the blue full line represents best fitting result. The hadron contamination is about $20 \%$ at $8 \mathrm{GeV}$, and decreases to lowest $1 \%$ at about $13 \mathrm{GeV}$ rapidly and slowly increase to $5 \%$ at $100 \mathrm{GeV}$.

\subsection{Secondary background}

The electron candidates we selected have two origins, one is from outer space (called primary), the other one is from the interaction of primary in the Earth's atmosphere (called secondary). At outer space, primary CREs follow approximate isotropic distribution. When entering the Earth's magnetic field, primary CREs always move long path and their original direction will be heavily deflected by geomagnetic force, while secondary CREs mainly generated near the Earth's atmosphere, making secondary CREs unable to move long-enough path in geomagnetic field. Consequently, space distribution of secondary CREs will not be effected by geomagnetic field significantly. As 
a result of this effect, primary CREs have very different angular distribution from secondary one. We can exploit a feature based on this phenomenon to estimate the fraction of secondary CREs. CREs' azimuth angle in the Earth centered coordinates can serve as this feature. The azimuth distribution of primary CREs can be obtained by IGRF model and particle tracing approach which are technically described in [2]. In this measurement, we used very similar method with Fermi-LAT, but update IGRF model from 11th generation to latest 12th generation which provide a definitive main field for epoch 2015, and a linear annual predictive secular model for 2015.0-2020.0. On the other hand, it is almost impossible to extract secondary's azimuth distribution feature by simulation because the path of "trapped" event cannot stand for real trajectory of secondary electron. In principle, we cannot acquire any primary CREs with energy less than cutoff if no secondary CREs exist. Thus, if we selected flight data event with energy $<<$ cutoff (i.e. $2 \mathrm{GeV}-4 \mathrm{GeV}$ ), it is reasonable to assume data sample are secondary CREs dominant and we can use this sample to extract secondary's azimuth distribution feature. The difference between secondary and primary CREs can be found in Fig3. When got these two features, we performed template fit to estimate the fraction of secondary and the result are shown in Fig3. From template fitting result, the secondary background ratio is about $56 \%$ below $10 \mathrm{GeV}$ and smoothly decreases to approximate 0 at about $12 \mathrm{GeV}$.

\section{Cosmic ray electrons spectrum and rigidity cutoff}

The spectrum is calculated by multiplying electron candidates count, the fraction of secondary and the ratio of hadron background and then dividing by the effective acceptance, width of energy bin and exposure time. The exposure time is determined by accumulating live time within every second in MaIlwain L bin [1,1.14], and live time is obtained by deducing dead time from total observation time. The spectrum is calculated in 30 energy bins shown in Fig4, the energy bin widths are chosen to be equal log value to make each bin satisfied with adequate and similar statistic. This kind of spectrum can be described by a parameterized formula. $d N / d E=c E^{\Gamma} /\left(1+(E / E c)^{-8}\right)$, where $\Gamma$ is the spectral index and Ec is rigidity cutoff for CREs. The fitting result shows that the rigidity cutoff in MacIlwain $\mathrm{L}$ range $[1,1.14]$ are $13.20 \mathrm{GeV}$ for data and $13.04 \mathrm{GeV}$ for MC back tracing. By directly comparing energy cutoff measured by flight data with the predicted one from MC back tracing, we found the absolute energy scale of BGO calorimeter is a little higher by a factor of $1.25 \%$. In the following section, we will study systematic uncertainty of this measurement.

\section{Systematic uncertainty analysis}

\section{1. binning $(0.15 \%)$}

In this measurement, to ensure bins have sufficient and similar statistic the energy widths of each bin $\Delta E$ are chosen to have equal log value. The migration from bin to its neighbor bins will induce an uncertainty on cosmic ray electron flux measurement and finally affect cut off energy. To estimate this kind of uncertainty, electron spectrum measured with AMS02 [4] is used to generate $1 \mathrm{M}$ electron sample and then energy resolution function of DAMPE extracted from beam test[1] is used to tune energy response in G4 simulation, then the matrix between primary energy and 


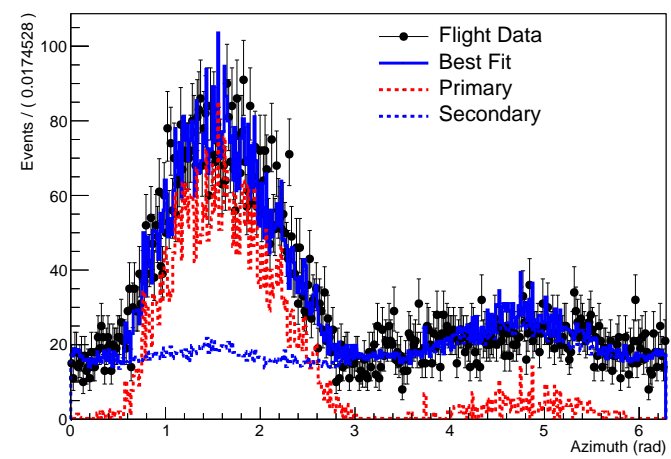

(a) $(8.0-10.3) \mathrm{GeV}$

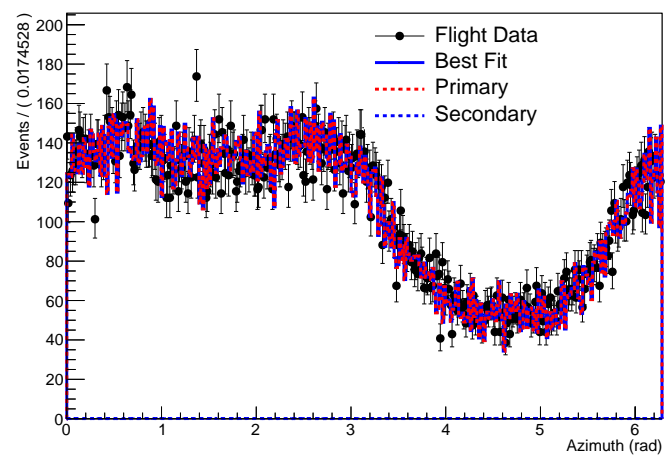

(c) $(14.4-15.7) \mathrm{GeV}$

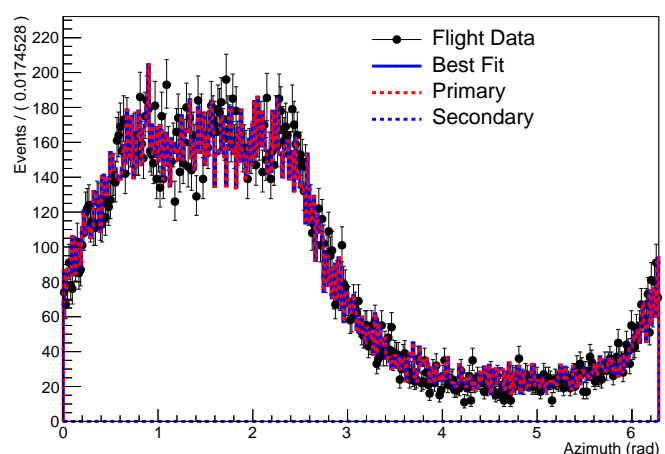

(b) $(12.1-13.2) \mathrm{GeV}$

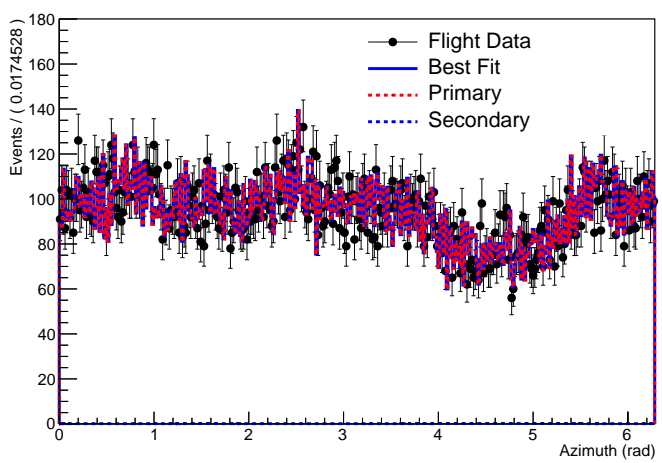

(d) $(17.1-18.6) \mathrm{GeV}$

Figure 3: Typical azimuth distribution at (a) $[8.0,10.3] \mathrm{GeV}$, (b) $[12.1,13.2] \mathrm{GeV}$, (c) $[14.4$, 15.7] GeV, (d) $[17.1,18.6] \mathrm{GeV}$ with McIlwain $\mathrm{L}$ at range $[1,1,14]$. There is an a well agreement between flight data and back tracing above $12 \mathrm{GeV}$ when secondary electron contamination is weak, which also demonstrates our particle tracing algorithm is reliable. As expected, azimuth distribution become flat with energy increase since geomagnetic force can't bend high rigidity electron so much.

deposited energy after correction was created and was used to unfolding CRE flux. The difference of cutoffs before unfolding and after unfolding is treated as uncertainty caused by binning. Thanks to the excellent energy resolution of DAMPE, it is found that the binning method uncertainty on cutoff energy is less than $0.15 \%$.

\section{2. choice of fitting range $(1.1 \%)$}

The energy range we selected to perform fitting can slightly affect fitting result value of the energy cutoff. To estimate the systematic uncertainty caused by choice of fitting range, we performed 500 fitting progresses with 500 different ranges which are wide or narrow but always cover cut off energy. And we take the RMS of fitting result values distribution as uncertainty induced by choice of fitting range. It is found to be less than $1.1 \%$ for this measurement.

\section{3. $\operatorname{IGRF}(0.5 \%)$}




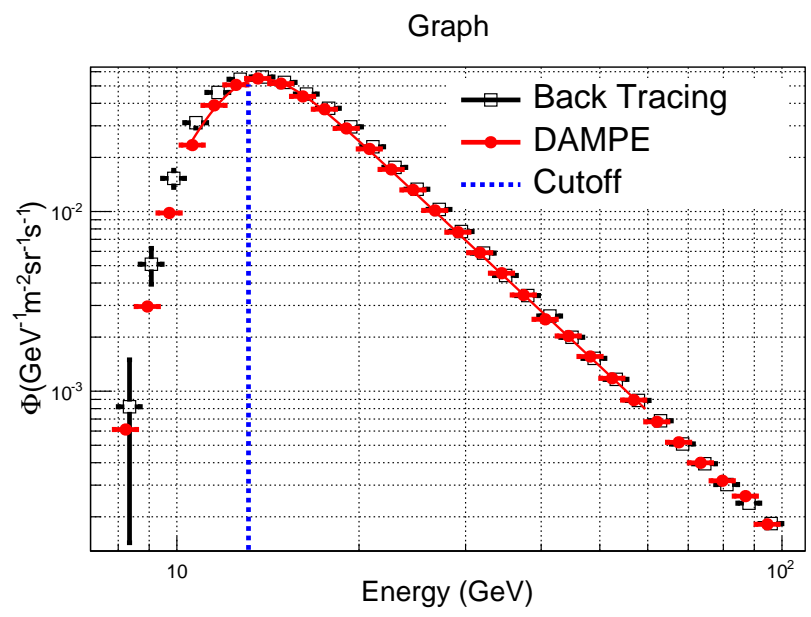

Figure 4: Cosmic ray electron flux at $1<L<1.14$. Red solid square is DAMPE flight data, black open square is back tracing results and red line is best fitting result for flight data. The errors on flux only contain statistic fluctuation and widths of error bars on energy only represent energy bins widths. Flight data agrees well with back tracing. By directly comparing fit results of cut off energy between flight data and backing tracing, absolute energy scale of DAMPE is higher by a factor of $1.23 \%$ than back tracing results.

The systematic error induced by IGRF model is assessed by directly comparing DAMPE result of Fermi-LAT result at same MacIlwain interval. We found that rigidity cutoff measured by DAMPE is lower than Fermi-LAT's value by $0.5 \%$.

\section{4. e/p Template $(0.4 \%)$}

The Monte Carlo template we used to estimate hadron background contamination could induce uncertainty on cutoff rigidity. From the Fig2, the background contamination are about $1 \%$ at $13 \mathrm{GeV}$. To estimate systematic error caused by template fitting, we smoothly verify the left tail of crystal ball function longer or shorter. We found that contamination changed within $0.5 \%$ at $13 \mathrm{GeV}$ and $1-2 \%$ at lower or higher energy range. By knowing flux uncertainty due to template, we randomly select 500 groups of flux to fit cutoff and find that systematic error due to MC template should be less than $0.4 \%$.

5. choice of energy interval for secondary template $(0.15 \%)$

Secondary template was obtained from low energy flight data sample $\left(E<<E_{\text {cutoff }}\right)$ where the CRE is secondary dominant. Choice of energy interval $(2-4 \mathrm{GeV})$ will affect shape of template and thus flux and final value of rigidity cutoff. To estimate this kind of uncertainty, we changed energy interval wider or narrower, closer or farther to the cutoff to extract template and do flux measurement and cutoff fitting. And we take the max deviation as the systematic uncertainty caused by secondary template. It is found that the uncertainty is about $0.15 \%$.

\section{Conclusion}

In this paper, we measured absolute energy scale of BGO calorimeter of DAMPE using 425 days flight data collected from 20160101 to 20170228 . By comparing geomagnetic cutoff on cos- 
mic ray electron and positron fluxes measured from data and back tracing, we found DAMPE's absolute energy scale greater than predicted by $1.25 \% \pm 1.75 \%$ (stat) $\pm 1.34 \%$ (sys) near $13 \mathrm{GeV}$.

\section{References}

[1] Zhiyong Zhang et al. The calibration and electron energy reconstruction of the BGO ECAL of the DAMPE detector, NIM A, Volume 836, 11 November 2016, Pages 98-104

[2] M. Ackermann et al. In-flight measurement of the absolute energy scale of the Fermi Large Area Telescope, Astroparticle Physics, volume 35, Pages,346 - 353, 2012

[3] Chuan Yue et al. A parameterized energy correction method for electromagnetic showers in BGO-ECAL of DAMPE, NIM A, Volume 856, 2017, Pages 11-16

[4] Aguilar, M. et al. Precision Measurement of the $\left(e^{+}+e^{-}\right)$Flux in Primary Cosmic Rays from 0.5 $\mathrm{GeV}$ to $1 \mathrm{TeV}$ with the Alpha Magnetic Spectrometer on the International Space Station, Phys. Rev. Lett. volume 113,issue 22,pages 221102,numpages 7, Nov 2014

[5] Carl E. EcIlwain, Coordinates for Mapping the Distribution of Magnetically Trapped Particles, Journal of Geophyiscs Research, volume 66,issue 11, Nov 1961

[6] Aguilar, M. et al. Precision Measurement of the Proton Flux in Primary Cosmic Rays from Rigidity 1 GV to 1.8 TV with the Alpha Magnetic Spectrometer on the International Space Station, Phys. Rev. Lett. volume 114,issue 17,pages 271103, numpages 19, Apr 2015

[7] M. Ackermann, et al. The Fermi Large Area Telescope on Orbit: Event Classification, Instrument Response Functions, and Calibration, The Astrophysical Journal Supplement Series, volume 203,number 1,pages 4, 2012

[8] D.F. Smart and M.A. Shea, A review of geomagnetic cutoff rigidities for earth-orbiting spacecraft, Advances in Space Research, volume 36,number 10, pages 2012 - 2020,2005 